\title{
Synchrotron oscillation effects on an rf-solenoid spin resonance
}

\author{
P. Benati, ${ }^{1}$ D. Chiladze, ${ }^{2,3}$ J. Dietrich, ${ }^{3}$ M. Gaisser, ${ }^{3}$ R. Gebel, ${ }^{3}$ G. Guidoboni, ${ }^{1}$ V. Hejny, ${ }^{3}$ A. Kacharava, ${ }^{3}$ V. Kamerdzhiev, ${ }^{3}$
} P. Kulessa, ${ }^{4}$ A. Lehrach, ${ }^{3}$ P. Lenisa, ${ }^{1}$ B. Lorentz, ${ }^{3}$ R. Maier,${ }^{3}$ D. Mchedlishvili, ${ }^{2,3}$ W. M. Morse, ${ }^{5}$ D. Öllers, ${ }^{1}$ A. Pesce, ${ }^{1}$ A. Polyanskiy, ${ }^{3,6}$ D. Prasuhn, ${ }^{3}$ F. Rathmann, ${ }^{3}$ Y. K. Semertzidis, ${ }^{5}$ E. J. Stephenson, ${ }^{7}$ H. Stockhorst,${ }^{3}$ H. Ströher, ${ }^{3}$ R. Talman, ${ }^{8}$ Yu. Valdau, ${ }^{9,10}$ Ch. Weidemann, ${ }^{1}$ and P. Wüstner ${ }^{11}$

${ }^{1}$ University of Ferrara and INFN, 44100 Ferrara, Italy

${ }^{2}$ High Energy Physics Institute, Tbilisi State University, 0218 Tbilisi, Georgia

${ }^{3}$ Institute für Kernphysik, Forschungszentrum Jülich, D-52425 Jülich, Germany

${ }^{4}$ Institute of Nuclear Physics PAN, 31-342 Krakow, Poland

${ }^{5}$ Brookhaven National Laboratory, Upton, New York 11973, USA

${ }^{6}$ Institute for Theoretical and Experimental Physics, 117259 Moscow, Russia

${ }^{7}$ Indiana University Center for Spacetime Symmetries, Indiana University, Bloomington, Indiana 47405, USA

${ }^{8}$ Cornell University, Ithaca, New York 14850, USA

${ }^{9}$ Petersburg Nuclear Physics Institute, 188300 Gatchina, Russia

${ }^{10}$ Helmholtz-Institut für Strahlen- und Kernphysik, Universität Bonn, Nussallee 14-16, D-53115 Bonn, Germany

${ }^{11}$ ZEL, Forschungszentrum Jülich, D-52425 Jülich, Germany

(Received 14 September 2012; published 19 December 2012)

\begin{abstract}
New measurements are reported for the time dependence of the vertical polarization of a $0.97 \mathrm{GeV} / \mathrm{c}$ deuteron beam circulating in a storage ring and perturbed by an rf solenoid. The storage ring is the cooler synchrotron (COSY) located at the Forschungszentrum Jülich. The beam polarization was measured continuously using a $1.5 \mathrm{~cm}$ thick carbon target located at the edge of the circulating deuteron beam and the scintillators of the EDDA detector. An rf solenoid mounted on the ring was used to generate fields at and near the frequency of the $1-G \gamma$ spin resonance. Measurements were made of the vertical beam polarization as a function of time with the operation of the rf solenoid in either fixed or continuously variable frequency mode. Using rf-solenoid strengths as large as $2.66 \times 10^{-5}$ revolutions/turn, slow oscillations $(\sim 1 \mathrm{~Hz})$ were observed in the vertical beam polarization. When the circulating beam was continuously electron cooled, these oscillations completely reversed the polarization and showed no sign of diminishing in amplitude. But for the uncooled beam, the oscillation amplitude was damped to nearly zero within a few seconds. A simple spin-tracking model without the details of the COSY ring lattice was successful in reproducing these oscillations and demonstrating the sensitivity of the damping to the magnitude of the synchrotron motion of the beam particles. The model demonstrates that the characteristic features of measurements made in the presence of large synchrotron oscillations are distinct from the features of such measurements when made off resonance. These data were collected in preparation for a study of the spin coherence time, a beam property that needs to become long to enable a search for an electric dipole moment using a storage ring.
\end{abstract}

DOI: 10.1103/PhysRevSTAB.15.124202

PACS numbers: 29.27.Bd, 29.20.db, 29.27.Hj, 41.75.Ak

\section{INTRODUCTION}

It has been proposed [1] that a storage ring can be used to search for an intrinsic electric dipole moment (EDM) on the particles in the circulating beam. That beam would be initially polarized along the direction of the beam velocity and held against precession in the ring plane by a special choice of beam conditions and ring electric and magnetic fields. The signal of an EDM would be the precession of the polarization from the longitudinal toward the vertical

Published by the American Physical Society under the terms of the Creative Commons Attribution 3.0 License. Further distribution of this work must maintain attribution to the author(s) and the published article's title, journal citation, and DOI. direction under the influence of the strong radial electric field pointing toward the center of the ring that exists in the beam frame of reference. This field, which bends the particle trajectories into a closed orbit, is present whether the ring is magnetic or electrostatic (as has been proposed for the proton case). However, it is first necessary to arrange the ring fields so that the precession of the spin relative to the velocity in the ring plane is suppressed. That precession (without the EDM component) is given by (with $\vec{\beta} \cdot \vec{B}=\vec{\beta} \cdot \vec{E}=0$ )

$$
\vec{\omega}=-\frac{q}{m}\left\{G \vec{B}+\left[G-\left(\frac{m}{p}\right)^{2}\right] \frac{\vec{\beta} \times \vec{E}}{c}\right\}
$$

where $G$ is the particle anomalous magnetic moment (also denoted by $a$ for leptonic anomalous moments). 
For protons with a positive anomalous magnetic moment, it is possible to make $\vec{\omega}=0$ when $\vec{B}=0$ (purely electrostatic bending elements) and $p=\frac{m}{\sqrt{G}}=0.701 \mathrm{GeV} / \mathrm{c}$. For deuterons with a negative anomalous magnetic moment, no such solution exists and the bending elements of the ring become a combination of vertical magnetic and outward radial electric fields such that the electric field is related to the magnetic field through

$$
E=\frac{G B c \beta \gamma^{2}}{1-G \beta^{2} \gamma^{2}} .
$$

In both cases, ring and experiment performance are enhanced by choosing as large an electric field as is practical.

At EDM sensitivity levels approaching $10^{-29} \mathrm{e} \cdot \mathrm{cm}$, the EDM signal would be a precession on the order of $10^{-5} \mathrm{rad}$ for a beam storage time of $20 \mathrm{~min}$. The observation of this small change in the vertical polarization component requires a beam polarimeter that is highly efficient and that has a large sensitivity to polarization. Such a polarimeter could rely mainly on the observation of scattered beam protons or deuterons from a carbon target, a process that has been used in similar applications for magnetic spectrometers with an efficiency of about $1 \%$ $[2,3]$. In addition, there must be methods to reduce and/ or correct systematic polarimeter errors under conditions where the beam-target geometry and instantaneous counting rate are changing. In a recent experiment using the scintillators of the EDDA detector [4,5] located at the cooler synchrotron (COSY) at the Forschungszentrum Jülich [6], a thick carbon target located at the edge of the circulating beam was used to demonstrate efficient operation, high spin sensitivity, and the ability to correct systematic errors at levels below $10^{-5}$ in the polarization measurement [7]. By arranging for a continuous, slow extraction of the beam onto the carbon block, this arrangement provided a means of continuously monitoring the beam polarization during the time that the beam was captured in the storage ring. This setup was used in the present study to observe deuteron polarizations while operating an rf solenoid on the ring. Such a continuous polarization monitor has not been available previously; its use makes available more information in a short period of experimental time compared to single point measurements based on inserting a carbon fiber into the beam [8].

An additional requirement for the EDM search is that the beam polarization remains large for the 20 min needed for the EDM effect to accumulate to a measurable level. Normally, momentum spread among the beam particles leads to differences in the precession rates given in Eq. (1) and the particle spins will decohere by spreading in the horizontal plane. The linear part of this effect may be canceled by using an rf cavity to bunch the beam, thereby imposing the same average cyclotron frequency on each particle. However, a lengthening of the orbital path that depends quadratically on the size of horizontal and vertical betatron oscillations while maintaining the cyclotron frequency will generate a smaller spread in spin precession rates that will lead to decoherence over a longer time. At this smaller level, there may also be a contribution that depends on the square of the deviation of the momentum from the central value. So a long-term goal of the EDM ring development program is to understand how to reduce or correct for such quadratic or higher order effects by, for example, adjusting the ring sextupole field components or cooling the beam.

In the first part of this development program, the plan was to use the rf solenoid located on the COSY ring to measure polarization changes in the vicinity of the $1-G \gamma$ resonance since the width of this resonance depends on the same betatron oscillations or momentum changes that lead to decoherence [8]. Model calculations of the vertical polarization led to the expectation that operation of the $\mathrm{rf}$ solenoid at a fixed frequency on or very close to the resonance would yield a series of damped polarization oscillations whose pattern was sensitive to the resonance width. A set of such measurements was completed with varying degrees of damping, but it was learned afterward that a much larger and different effect was at work, created by an interplay between the rf solenoid and synchrotron oscillations within the bunched beam. This paper describes that effect and how its characteristics make it different from the effects of momentum spread on resonance width.

The studies reported here made use of a polarized deuteron beam circulating at a momentum of $p=$ $0.97 \mathrm{GeV} / \mathrm{c}$, a value chosen to optimize the analyzing power of elastic scattering events recorded in the EDDA detector. At this momentum it was also possible to electron cool the bunched beam. Recording data with and without electron cooling made drastic differences in the results, a feature that was crucial in identifying the mechanisms at work in the ring. With electron cooling turned on, the beam was gathered within tens of seconds into a bunch a few meters in length (out of a circumference of $183 \mathrm{~m}$ ) with a correspondingly small momentum spread. Once the beam was cooled, continuous operation of the rf solenoid on the spin resonance resulted in vertical polarizations that oscillated between (nearly) 1 and -1 with no indication of damping for times up to one minute. These oscillations are consistent with a simple model of the process that contains two spin rotations, one for all of the bending magnets in the ring and another for the rf solenoid. No further details of the machine lattice are needed for a description of these data. Off resonance, the oscillations persist, but with a smaller amplitude, a higher frequency, and an oscillation range that remains close to 1 . These effects are consistent with a mismatch in the frequencies of the rf solenoid and the $1-G \gamma$ resonance and with the features found in prior experiments [8].

Without electron cooling in operation, the beam spreads longitudinally until there is a significant particle 
population for at least half of the ring circumference, as the beam was bunched at the first harmonic. Particles oscillated from one end of this bunch to the other at a synchrotron frequency of $331 \mathrm{~Hz}$. In doing so they changed their timing with respect to the phase of the oscillating rfsolenoid field. With a cyclotron frequency of $750 \mathrm{kHz}$, the rf solenoid was operated at $f_{\text {CYC }}(1-G \gamma)=871 \mathrm{kHz}$ (see a later discussion of Table IV), a value above the cyclotron frequency that allowed a large phase change during the course of a single synchrotron oscillation. This effectively weakened the ability of the rf solenoid to precess the polarization, creating a part of the beam distribution whose vertical polarization was oscillating at a slower frequency. The combination of all beam particles then followed a damped oscillation with characteristics different from those found for off-resonance behavior. Damping took place early in the pattern and was centered close to zero vertical polarization. These features were also described within the two rotation model, but only if the timing of synchrotron oscillations relative to the rf solenoid was included. The results proved to be very sensitive to the details of the particle distribution within the beam bunch, and could be used to unfold that distribution.

Sections II and III of this paper describe the experimental setup of the EDDA detectors and the data acquisition system. As a means of constraining the model, measurements were also made of the beam and machine properties, as discussed in Sec. IV. Section V summarizes the "no lattice" spin-tracking model. The parts of Sec. VI describe the effects of synchrotron oscillations in detail, with a conclusion given in Sec. VII.

\section{EXPERIMENTAL SETUP}

The polarized deuteron beam was produced in an atomic beam source [9] with two stages of sextupole separator magnets, each followed by rf transition units. This provided for several combinations of vector $\left(p_{\mathrm{V}}\right)$ and tensor $\left(p_{\mathrm{T}}\right)$ polarization. The polarization states chosen for this experiment included two with only vector polarization, two with a combination of both vector and tensor polarization, and one unpolarized. A cyclotron was used to raise the beam energy to $76 \mathrm{MeV}$ for injection into the COSY storage ring. On the injection beam line there is a scattering chamber with a carbon rod target. Deuterons elastically scattered from the rod were counted by setting a pulse height threshold on the signals from silicon detectors mounted symmetrically in the low-energy polarimeter on the left and right sides of the beam. Based on an interpolation of existing deuteron-carbon elastic scattering analyzing powers [10,11], the analyzing power of this system was $A_{Y}=0.65(0.64) \pm 0.04$ at a laboratory angle of $50^{\circ}$ $\left(45.9^{\circ}\right)$ where the detectors were placed at different times during the experiment. The calibration error was taken from Ref. [5].
TABLE I. Vector polarization $p_{\mathrm{Y}}$ before and after ion source change.

\begin{tabular}{lcrr}
\hline \hline Type & $p_{\mathrm{Y}}$ sign & Before & \multicolumn{1}{c}{ After } \\
\hline Vector & + & $0.47(3)$ & $0.42(3)$ \\
& - & $-0.38(3)$ & $-0.37(3)$ \\
Tensor/vector & + & $0.62(4)$ & $0.56(4)$ \\
& - & $-0.34(2)$ & $-0.33(2)$ \\
\hline \hline
\end{tabular}

The detector angle change also corresponded in time to a change in the operating condition of the polarized ion source to optimize transmission through the cyclotron. The values of the vector polarization $p_{\mathrm{Y}}$ are given in Table I before and after the change. The errors reflect the scale errors of the calibration.

One concept for the EDM polarimeter involves stopping detectors that deliver their largest signals for elastic scattering events. Reactions that produce lower energy particles, usually a deuteron or a proton, are removed with an absorbing medium between the target and the detector. A similar arrangement was achieved in a previous experiment [7] using the scintillators of the EDDA detector [4,5]. Long scintillators, called "bars," ran parallel to the beam and were read out with photomultiplier tubes mounted on the downstream end. These 32 scintillators were divided into groups of eight, corresponding to scattering to the left, right, down, or up directions. Outside the bars were "rings" that intercepted particles scattering through a range of polar angles beginning at $9.1^{\circ}$. During gain calibration of the ring detectors, elastic events were clearly evident starting in the third ring at angles greater than $10.9^{\circ}$. Four consecutive EDDA rings were included in the "polarimeter group," extending the sensitive angle range to $21.5^{\circ}$. Over this angle range the vector analyzing power for the elastic scattering of deuterons from carbon is positive and passes through the first interference maximum [12], making this an excellent range for operation as a polarimeter. The energy loss through the target material, beam pipe, and an absorbing tube removed much unwanted flux, especially protons from deuteron breakup. But there still remained some flux from inelastic scattering and $(d, p)$ reactions with low $\mathrm{Q}$-values that reduced the average analyzing power.

The scattering target for the polarimeter was a carbon tube $15 \mathrm{~mm}$ long that surrounded the beam [7]. Slow extraction of the beam onto the target was achieved by locally steering the beam vertically upward into the top edge of the tube. Deuterons intercepting the target front face pass through the full target thickness, which greatly increases their probability of scattering into the EDDA scintillator system. The efficiency for observing an event that was subsequently used to calculate a polarization relative to the number of deuterons lost from the beam was about $4.4 \times 10^{-4}$ for much of the experiment, but fell by over a factor of 2 as beam steering changed. 
The requirement that elastically scattered deuterons stop within the forward angle ring detectors led to the choice of $0.97 \mathrm{GeV} / \mathrm{c}$ as the optimum beam momentum.

The ring and bar photomultiplier signals for each quadrant of the EDDA detector (left, right, down, or up) were summed into a single analog signal and counted by a scaler based on a common pulse height threshold for the four quadrants. The threshold was set to the maximum allowed for the module $(-125 \mathrm{mV})$. This made the trigger rates similar $( \pm 7 \%$ for the unpolarized beam) for all four quadrants. The analyzing power was $A_{Y}=0.32 \pm 0.02$ with the error dominated by the calibration of the lowenergy polarimeter. The scalers were read out 400 times per second. When the observed polarization changes were slow enough to not require this many time bins, the bins were combined into groups for better statistics on the asymmetry data points. This polarimeter scheme offers a continuous monitor of the polarization during the beam store in contrast to previous experiments where observations were made at only one time during the polarization manipulation process [8].

\section{VERTICAL POLARIZATION MEASUREMENTS}

The triggers for the four segments of the EDDA detector (left, right, down, and up) were recorded in a single computer file for each run. A run consisted of a number of stores whose events could be added as a function of time since the start was synchronized to polarization precession operations through the use of a reproducible start time marker. The four polarization states listed previously as well as an unpolarized state were loaded sequentially for each new store with the series continuing until the last store of the run was ended.

Positive and negative vector polarizations were available with the two vector polarized states or the two states containing a combination of vector and tensor polarization. In each of these cases, the vector asymmetry was determined from the "cross ratio" [13] given by Eq. (3) as

$$
\epsilon=\frac{r-1}{r+1}, \quad \text { where } r^{2}=\frac{L(+) R(-)}{L(-) R(+)}
$$

and $L$ and $R$ are the count rates for the left and right systems for the positive $(+)$ and negative $(-)$ polarization states. This combination of count rates tends to suppress errors that arise as first-order contributions from geometric misalignments due to beam position or angle, or from detector acceptance. During the run, a more extensive calibration of the polarimeter sensitivity to systematic geometric and rate problems was conducted in the manner proscribed in Brantjes et al. [7]. Subsequent investigation showed that systematic errors from the use of the cross ratio formula were typically much less than $10^{-4}$. Thus, no further corrections were included in the work reported here.
Analysis of the two vector polarized states and the two vector/tensor polarized states produced two cross ratio asymmetries. For comparison to the model calculations to be discussed later, the two sets of cross ratio data from each run were normalized to one based on the asymmetries recorded prior to any polarization manipulations being made. Then the two measurements were averaged. This combined all of the polarized beam data from a given run into one time-dependent set of vector polarization measurements. The asymmetry from the tensor components was calculated, but the tensor analyzing power for this particular setup of the EDDA scintillators and thresholds proved to have a very small value and this asymmetry was never used. All of the polarization manipulation processes involved the use of an rf solenoid located in the COSY ring where $t=0$ represented the start of the field ramp-up for the solenoid.

\section{ACCELERATOR PARAMETERS}

The momentum for this experiment, $0.97 \mathrm{GeV} / \mathrm{c}$, was within the range where it was possible to electron cool the beam. Expecting that we would want to avoid a large spin decoherence due to momentum spread in the beam, all measurements were made with the beam bunched. The most significant comparison became the difference between the data features for the cooled and uncooled beam. In order to have information available for modeling the results, a number of machine parameters were measured for the various running conditions. These are summarized here.

The horizontal and vertical betatron tunes were $Q_{\mathrm{X}}=$ 3.60 and $Q_{\mathrm{Y}}=3.62$.

The momentum compaction factor $\alpha$, defined as the fractional change in the orbit length for a given fractional change in the momentum,

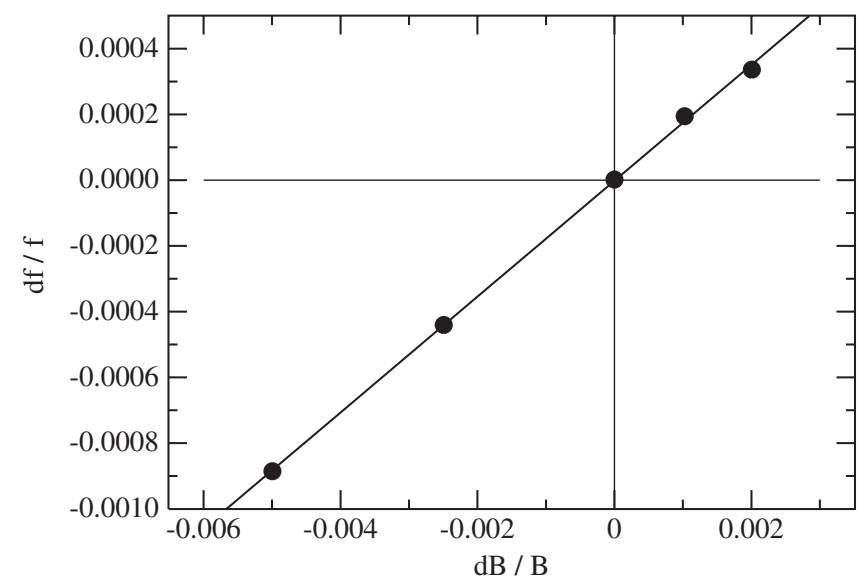

FIG. 1. Measurements of the fractional change in the cyclotron frequency as a function of the fractional change in the magnetic field. The best fit slope is a measure of the momentum compaction factor. 
TABLE II. Machine properties.

\begin{tabular}{lcc}
\hline \hline Item & Uncooled & Cooled \\
\hline$\Delta p / p$ & $8.02 \pm 0.23 \times 10^{-4}$ & $4.91 \pm 0.13 \times 10^{-5}$ \\
Width, $\Delta \mathrm{X}$ & $6.01 \mathrm{~mm}$ & $1.43 \mathrm{~mm}$ \\
Width, $\Delta \mathrm{Y}$ & $6.51 \mathrm{~mm}$ & $1.77 \mathrm{~mm}$ \\
Emittance, $\epsilon_{\mathrm{X}}$ & $1.6 \mu \mathrm{m}$ & $0.09 \mu \mathrm{m}$ \\
Emittance, $\epsilon_{\mathrm{Y}}$ & $5.8 \mu \mathrm{m}$ & $0.42 \mu \mathrm{m}$ \\
\hline \hline
\end{tabular}

$$
\alpha=\frac{\Delta L / L}{\Delta p / p}=\frac{\Delta f / f}{\Delta B / B}=0.177 \pm 0.003,
$$

was measured by recording the change in the orbit frequency for a small change in the ring magnetic fields, as shown in Fig. 1. The value is listed in Eq. (4). From this, it is possible to calculate the slip factor as

$$
\eta=\frac{\Delta f / f}{\Delta p / p}=\alpha-\frac{1}{\gamma^{2}}=\frac{1}{\gamma_{\mathrm{tr}}^{2}}-\frac{1}{\gamma^{2}}=-0.612 \pm 0.003 .
$$

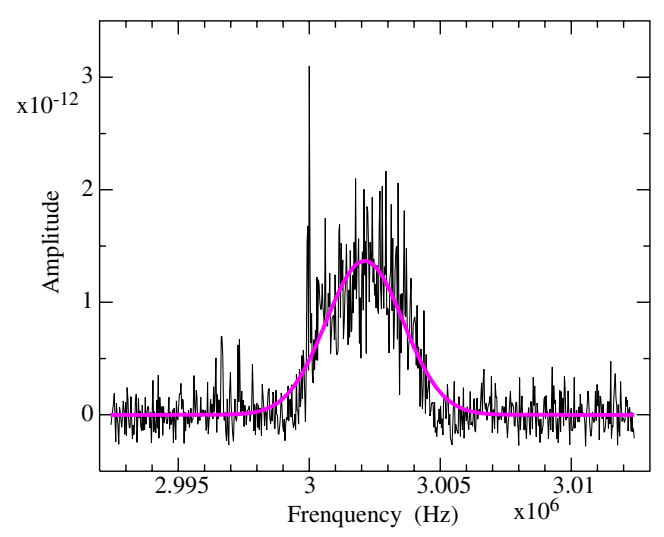

FIG. 2. Measurements of the Schottky signal on the fourth harmonic for an uncooled beam. The frequency is nominally $3002410 \mathrm{~Hz}$. The Gaussian fit is shown by the purple curve.

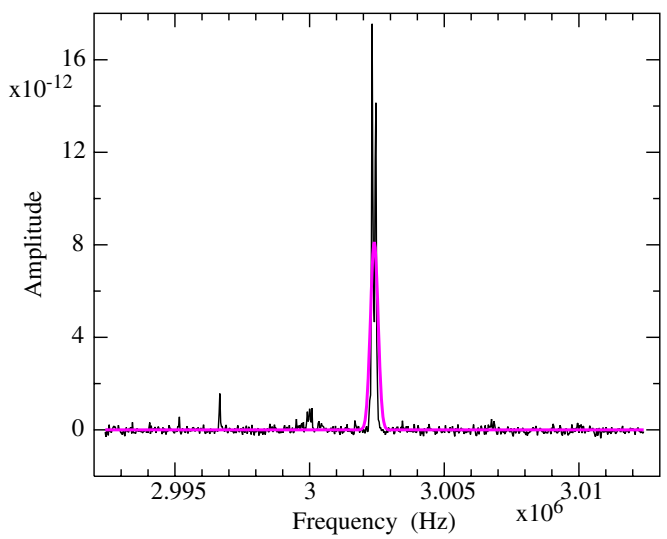

FIG. 3. Measurements of the Schottky signal on the fourth harmonic for a cooled beam. The Gaussian fit is shown by the purple curve.
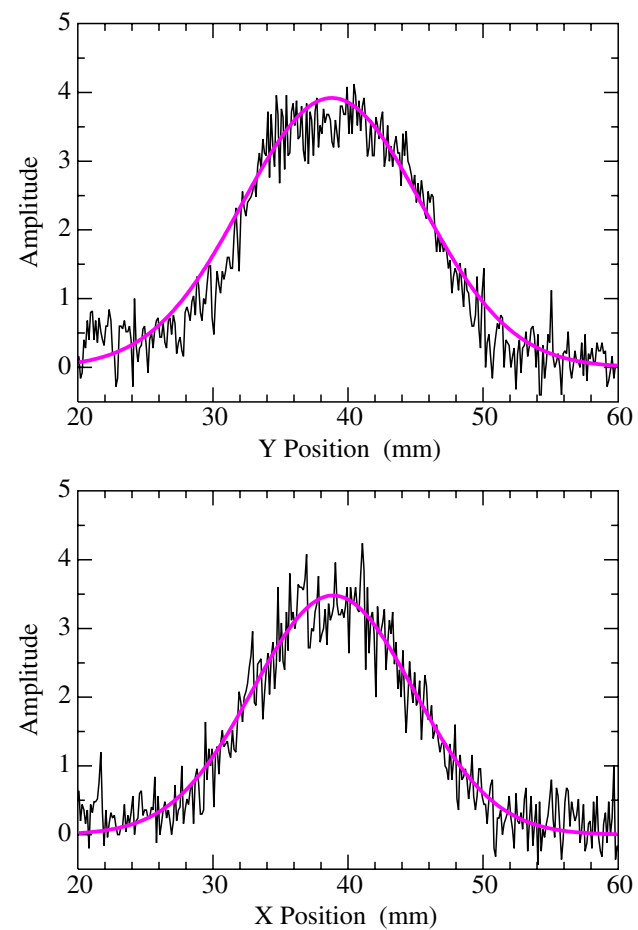

FIG. 4. Measurements of the vertical (top) and horizontal (bottom) beam profiles for an uncooled beam. The purple curves represent Gaussian fits to the profile shape.
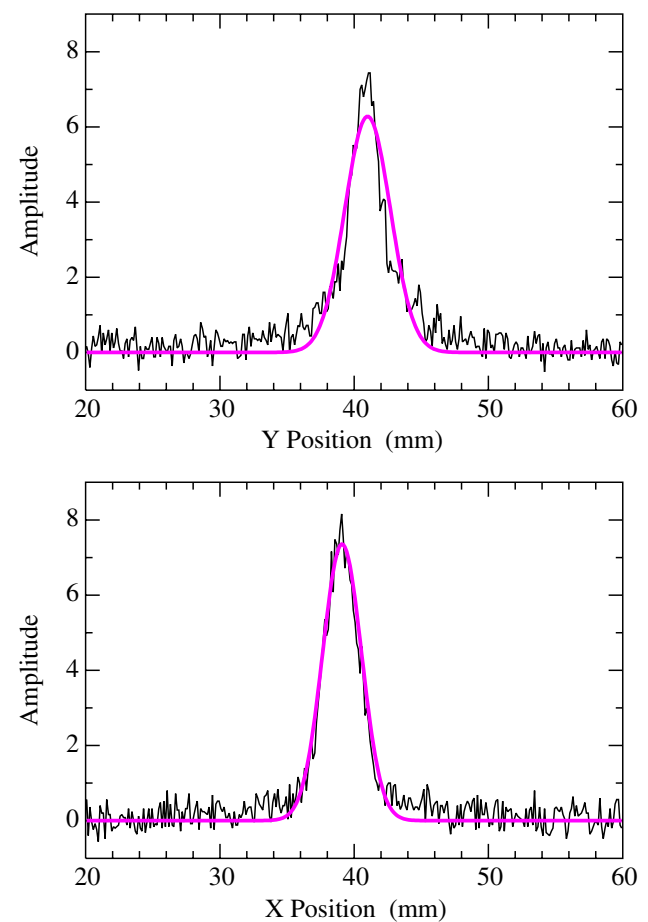

FIG. 5. Measurements of the vertical (top) and horizontal (bottom) beam profiles for a cooled beam. The purple curves represent Gaussian fits to the profile shape. 


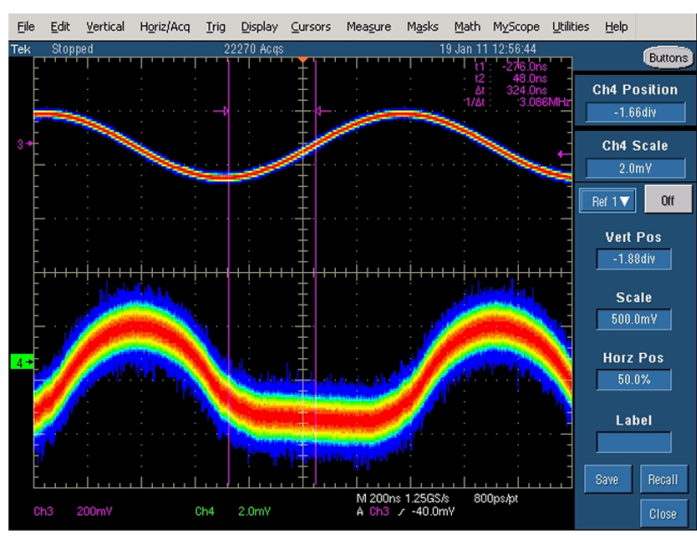

FIG. 6. Oscilloscope traces of the main rf (top) and beam pickup (bottom) for the uncooled beam. The time trace represents roughly $1.5 \mathrm{rf}$ periods. Note that the pickup sees no beam for about half of the machine cycle.

Other parameters measured separately for cooled and uncooled beams are given in Table II. The momentum spread was obtained from a fit of a Gaussian shape to the Schottky frequency distribution measured on the fourth harmonic of the cyclotron frequency after division by the slip factor (see Figs. 2 and 3).

The size of the horizontal and vertical emittances was obtained by first measuring the beam profile through an observation of residual gas ionized by the passage of the beam. Once recorded, these profiles were reproduced with a Gaussian function. Examples of these profiles and the fits are shown in Figs. 4 and 5. The Gaussian widths are given in Table II. The beta functions for the lattice were generated by MAD. For the position of the profile monitor they are $\beta_{\mathrm{X}}=22.12 \mathrm{~m}$ and $\beta_{\mathrm{Y}}=7.32 \mathrm{~m}$. From these values we obtained the emittances shown in Table II.

The beam was bunched on the first harmonic $(h=1)$ with a maximum oscillator voltage of $400 \mathrm{~V}$. For the uncooled beam, this captures most of the beam into about half of the ring circumference, as illustrated in Fig. 6.

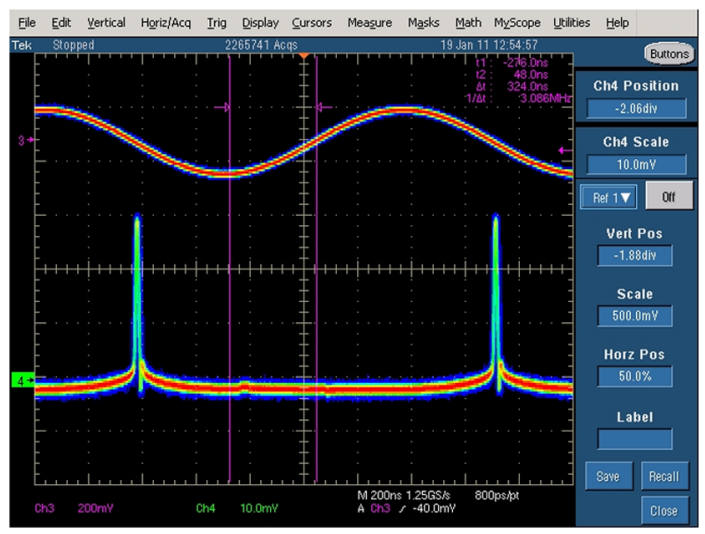

FIG. 7. Like Fig. 6 except for being a cooled beam. Most of the beam has been gathered into a narrow peak. Note the presence of a long residual uncooled tail on the sides.
When electron cooling is applied at the beginning of beam storage, the momentum spread of the beam is greatly reduced as noted above. This is illustrated by the correspondingly smaller geometrical size of the cooled beam shown in Fig. 7. The cyclotron frequency was $750602.5 \mathrm{~Hz}$.

\section{SPIN-TRACKING MODEL}

The precession of the deuteron spins under the influence of magnetic fields in the ring can, in many cases, be considered broadly without the need to include explicitly the contribution of each magnetic device located along the ring. For the data reported here, such a "no lattice" model can be reduced to two rotations that apply for each turn of the beam around the ring. One is a rotation of spins around the $\hat{y}$ axis perpendicular to the ring plane that arises from the cumulative effect of the ring dipoles. The precession angle on each turn is given by the size of the anomalous part of the deuteron magnetic moment, $G=(g-2) / 2$. This rotation in radians is

$$
\omega_{a}=2 \pi \nu_{\mathrm{S}}=2 \pi G \gamma,
$$

where $\nu_{\mathrm{S}}=G \gamma$ is the spin tune. It is sufficient to write the spin rotations in the classical form of a macroscopic magnetic dipole in an external magnetic field as it alters the spin vector $\vec{S}$ :

$$
\vec{S}^{\prime}=\left(\begin{array}{ccc}
\cos \omega_{a} & 0 & -\sin \omega_{a} \\
0 & 1 & 0 \\
\sin \omega_{a} & 0 & \cos \omega_{a}
\end{array}\right) \vec{S} .
$$

The rotation angle, $\omega_{a}$, is nearly the same for all of the deuterons in the beam and nearly the same across time; it changes only as $\gamma$ changes.

The second rotation is generated by an rf solenoid. It presents an oscillating field where the angle of spin rotation is given by

$$
\begin{aligned}
\theta_{\mathrm{S}} & =2 \pi \epsilon \cos \left(\omega_{\mathrm{S}} t\right), \\
\omega_{\mathrm{S}} & =2 \pi\left[f_{\mathrm{CYC}}(1-G \gamma)+\delta(t)\right],
\end{aligned}
$$

where $t=n / f_{\mathrm{CYC}}$ with $n$ the turn number. The $1-G \gamma$ harmonic of the spin tune $G \gamma$ was chosen before the experiment to best match the peak power output of the solenoid's driver amplifier. $\delta(t)$ represents a small perturbation that may or may not be time dependent and that is chosen in the design of the experiment to study phenomena near the resonance. The strength of the rf solenoid is given by $\epsilon$. The rf solenoid rotates the spin about the beam, or $\hat{z}$, direction. This rotation is described by

$$
\vec{S}^{\prime}=\left(\begin{array}{ccc}
\cos \theta_{\mathrm{S}} & -\sin \theta_{\mathrm{S}} & 0 \\
\sin \theta_{\mathrm{S}} & \cos \theta_{\mathrm{S}} & 0 \\
0 & 0 & 1
\end{array}\right) \vec{S} .
$$


Repeated applications of these two rotations for each turn of the beam around the ring generates $\vec{S}(t)$ from a given starting point $\vec{S}(0)$.

The synchrotron oscillations were taken to be longitudinal sinusoidal oscillations in the $Z$ direction (along the beam) about the center of the beam bunch with amplitude $A$ and a frequency of $331 \mathrm{~Hz}$ as given by

$$
z(t)=A \sin \left(2 \pi f_{\mathrm{SYNC}} t\right) .
$$

Synchrotron oscillations are also represented as a phase, $\phi$, where $\phi(t)=2 \pi z(t) / C$ and $C$ is the ring circumference. This relationship assumes operation of the storage ring on the first harmonic.

At the same time there is an oscillation of the momentum whose change, $\Delta p / p$, is $90^{\circ}$ out of phase with the position oscillation,

$$
\frac{\Delta p}{p}(t)=\frac{1}{\eta} \frac{f_{\mathrm{SYNC}}}{f_{\mathrm{CYC}}} 2 \pi \frac{A}{C} \cos \left(2 \pi f_{\mathrm{SYNC}} t\right)
$$

where $C$ is the ring circumference and $\eta$ is the slip factor. Changes to the spin tune are scaled by

$$
\frac{\Delta \nu_{s}}{\nu_{s}}=\beta^{2} \frac{\Delta p}{p} .
$$

The precession of the spin as it goes around the ring is changed because the momentum has changed, so that

$$
\omega_{a}=2 \pi\left(\nu_{s}+\Delta \nu_{S}\right)
$$

The main effect that is described in the paper arises because the phase of the rf solenoid as the particle passes through it has changed so that the rotation is for a different transit time

$$
\begin{aligned}
\theta_{\mathrm{S}}= & 2 \pi \epsilon \cos \left(2 \pi f_{\mathrm{CYC}}(1-G \gamma)\right. \\
& \left.\times\left[t-\frac{A}{f_{\mathrm{CYC}} C} \sin \left(2 \pi f_{\mathrm{SYNC}} t\right)\right]+\delta(t)\right) .
\end{aligned}
$$

As will be covered in the next section, detailed agreement with the uncooled data was made possible by choosing the appropriate distribution of orbit amplitudes $A$.

The sine and cosine functions used here to describe the synchrotron motion represent the solution for the differential equation of simple harmonic motion in a parabolic potential. A more accurate model would involve a sinusoidal potential and numerical integration of the synchrotron motion. This was tested for the present situation. While this changes some details of the shape of the beam distributions described later, there is no essential change in either the quality of the reproduction of the measurements or the interpretation based on this model.

\section{DATA AND INTERPRETATION}

\section{A. Determining model parameters}

The critical parameters for the model were determined from comparisons to key pieces of data. These parameters included the frequency of the $1-G \gamma$ resonance and the magnetic field strength of the rf solenoid for a given setting of the power controls. The broad distribution of amplitudes that make up the synchrotron oscillations within the beam bunch (shown in Fig. 6) also proved to be critical, but only for the uncooled measurements. Once these were known, a variety of effects could be successfully modeled. All curves shown in subsequent figures were made using the model described in the last section.

The frequency of the $1-G \gamma$ resonance was estimated at the beginning of the experiment by making a FroissartStora frequency sweep [14] across the expected location of the resonance using the uncooled beam. The frequency at which the polarization changed sign was taken as the initial resonance location. Figure 8 shows the data from this scan, which was started at $871200 \mathrm{~Hz}$ (at a time of $5.8 \mathrm{~s}$ ) and ramped at a speed of $10 \mathrm{~Hz} / \mathrm{s}$ for a total of $400 \mathrm{~Hz}$. The zero crossing is clearly evident near $29 \mathrm{~s}$. The polarization does not completely reverse, an issue that we will discuss later as a consequence of synchrotron oscillations rather than a ratio of rf-solenoid strength to ramping speed that is too small.

Refinements to the position of the resonance were made using the rf solenoid operating at a fixed frequency in the immediate neighborhood of the resonance. Being off resonance by even a fraction of one $\mathrm{Hz}$ creates clear changes in the polarization oscillation pattern and the final

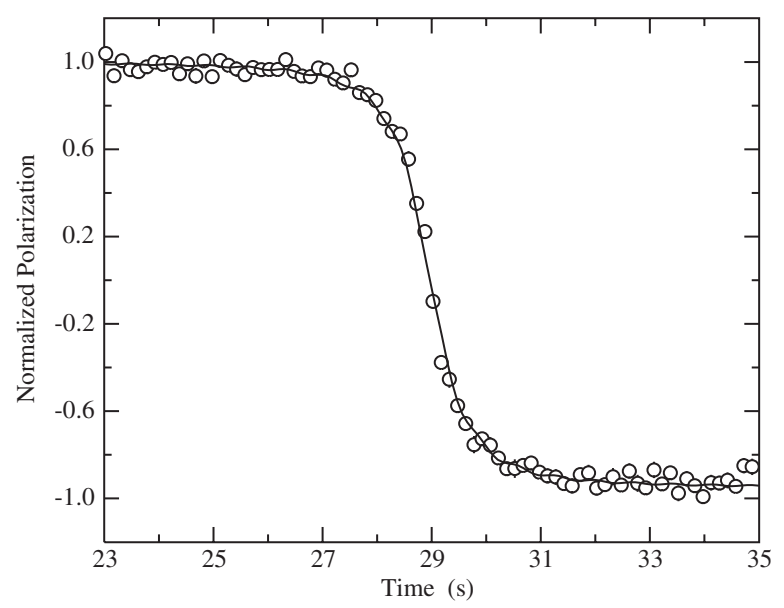

FIG. 8. Froissart-Stora scan across the $1-G \gamma$ resonance, which is close to the point where the polarization passes through zero. These data were measured with the uncooled beam. The curve is calculated assuming a resonance frequency of $871434 \mathrm{~Hz}$ and a ramp rate of $40 \mathrm{~Hz} / \mathrm{s}$ starting from $871200 \mathrm{~Hz}$ at 5.8 s. Some tracks with large synchrotron amplitudes have been removed to achieve agreement with the final polarization. 
polarization after the oscillations are damped (in the uncooled case). If these changes are matched by model calculations where the separation from the resonance is adjusted, then it becomes possible to locate the resonance with a precision of about $0.1 \mathrm{~Hz}$. The most precise results were obtained with the cooled beam. In this case the polarization oscillations persist for a long time and a precise measurement is possible of their frequency. From this frequency, measured with the rf solenoid on resonance, it is also possible to deduce the effective strength of the rf solenoid. Since the measurement of the resonance position and the solenoid strength utilize the same data, we will summarize our results using the data from the cooled beam.

The cooled beam measurements on resonance are shown in Fig. 9. The data represent a slow oscillation of the vertical component of the polarization with a period of about $2 / 3 \mathrm{~s}$. While the magnitude of the oscillation is somewhat less than one, it persists for the $55 \mathrm{~s}$ that the rf solenoid was kept running after the initial ramp-up, which
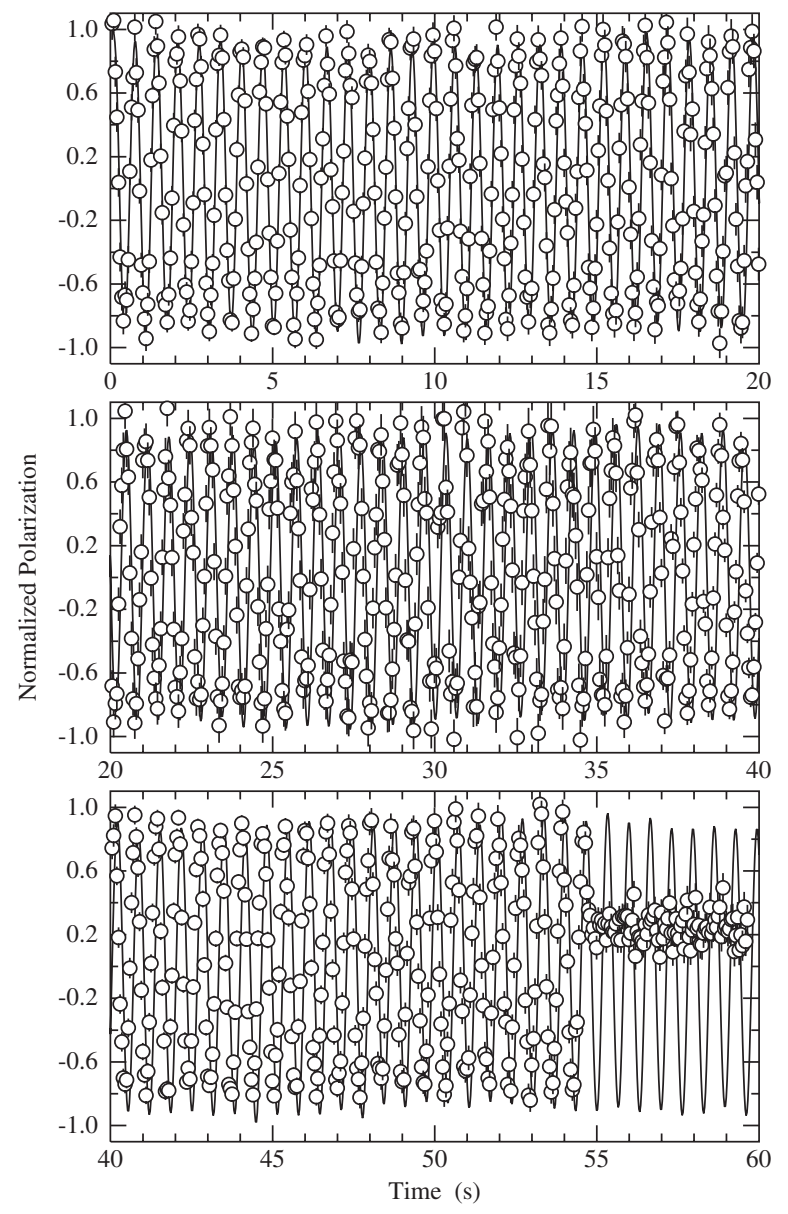

FIG. 9. Measurements made with a cooled beam and the rfsolenoid operating on resonance with a strength of $4.05 \times$ $10^{-6} \mathrm{rev} /$ turn. The curve is a model calculation. These data were taken with the rf solenoid operating at $871434 \mathrm{~Hz}$. The rf solenoid is turned off just before $t=55 \mathrm{~s}$. lasted $200 \mathrm{~ms}$. This permits a very precise determination of the magnetic field strength of the rf solenoid by matching the frequency of the polarization oscillation using calculations with the model described in the previous section. The oscillation pattern requires an effective strength of $\epsilon=4.05 \pm 0.01 \times 10^{-6} \mathrm{rev} /$ turn. The reduction in polarization is best understood as a result of an uncooled component in the beam whose distribution of synchrotron amplitudes is similar to that for the completely uncooled beam. This effect will be reviewed later. So long as the rfsolenoid frequency remain synchronized to the precession of the horizontal component of the polarization due to the ring dipoles (thus giving rise to the spin tune), then these oscillations will persist. (The depolarizing effects of either finite beam emittance or the quadratic momentum spread are suppressed for reasons that go beyond the scope of this paper and will be described elsewhere.)

If the rf solenoid is operated at a fixed frequency away from the center of the resonance, then the oscillation pattern quickly changes. If the difference is, for example, $1 \mathrm{~Hz}$, then the solenoid will get out of phase with the horizontal precession by $90^{\circ}$ in the first $0.25 \mathrm{~s}$. At this time the rf-solenoid rotations will be acting at a right angle to the angle that takes the polarization away from the vertical axis. No progress will be made toward changing the vertical component of the polarization, and the downward motion of the polarization will come to a halt. At later times as the phase slip moves toward $180^{\circ}$, the rf solenoid will be acting to move the polarization back toward the positive $\mathrm{Y}$ axis. When the phase slip is $180^{\circ}$, then the vertical polarization will again be +1 . For the next half second, this process is repeated. These effects reduce the magnitude of the oscillations even as the maximum polarization remains nearly equal to one. The change in magnitude is related to an increase in the frequency of oscillation, which can in turn be related to the difference in the frequencies of the rf solenoid and the polarization precession.

With very small changes being made in the rf-solenoid frequency $( \pm 1$ and $\pm 2 \mathrm{~Hz}$ ), it was assumed that the rfsolenoid strength was unchanged after the data taken in Fig. 10. Then the only parameter that may be adjusted in order to match the oscillation frequency is the frequency separation between the rf solenoid and the resonance. The shifts needed for the off-resonance data are given in Table III, along with the average and an error that spans the four cases of Fig. 10. Since the additional frequency shifts move the points below the resonance away from the resonance, and the point above the resonance toward the resonance, the resonance frequency is higher (see caption to Fig. 8) at $871434.13 \pm 0.04 \mathrm{~Hz}$.

The data and calculations of Fig. 10 illustrate the general features of the vertical polarization when operating the $\mathrm{rf}$ solenoid off resonance. As the distance from the resonance increases, the oscillation frequency increases, as is shown 


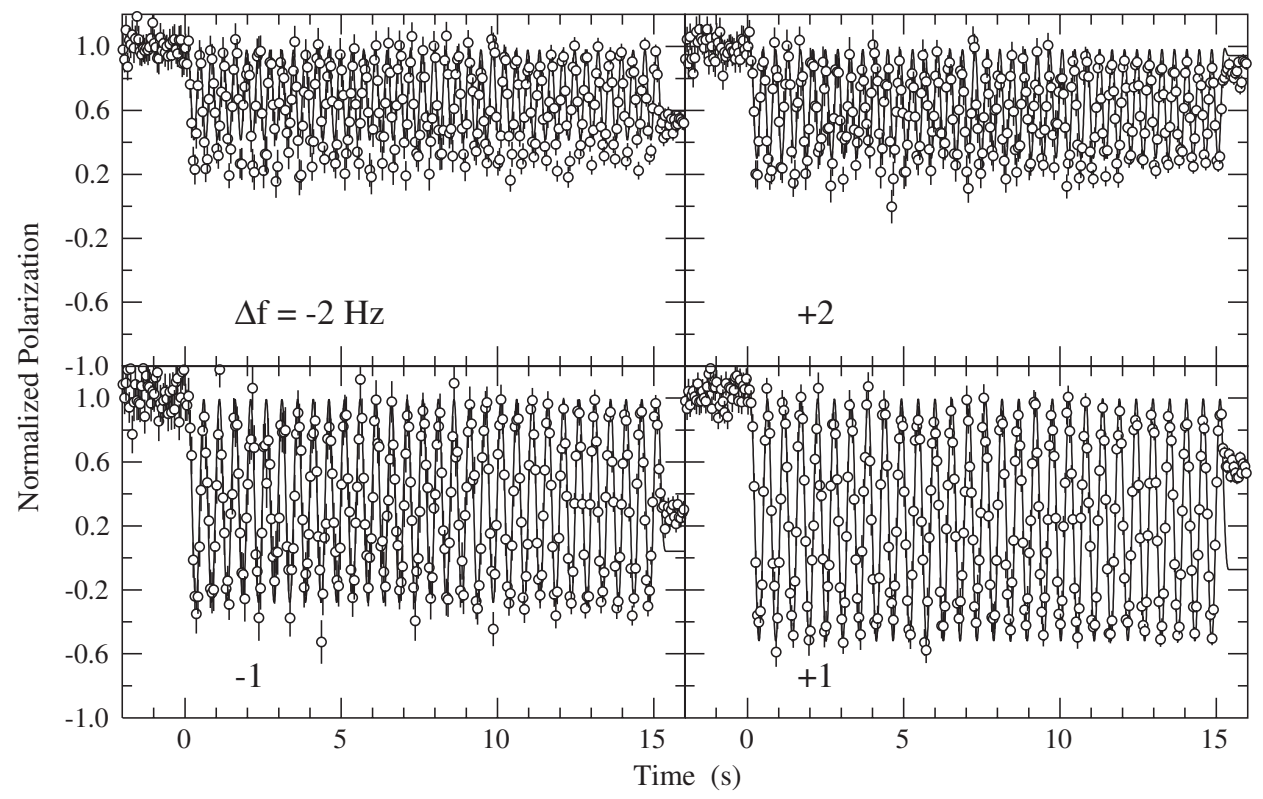

FIG. 10. Measurements of the vertical component of the polarization (normalized to one at the beginning of the process) with the rf-solenoid frequency displaced from the nominal resonance frequency of $871434 \mathrm{~Hz}$ by an amount $\Delta f$ as shown in each panel. The rf solenoid was on for $15 \mathrm{~s}$ and then turned off. The rf solenoid operated at a strength of $\epsilon=4.43 \times 10^{-6} \mathrm{rev} / \mathrm{turn}$ (higher than for the data for Fig. 9 because of a change in the rf power). The line represents a calculation made to match the frequency of the polarization oscillations by changing the value of $\Delta f$ by a fraction of one $\mathrm{Hz}$ (see Table III). The calculations involved only one particle track and no synchrotron oscillations.

in the right-hand column of Table III and Fig. 10. At the same time the oscillation amplitude goes down. Note, in particular, that the top of the oscillation pattern always remains close to one so that off-resonance polarization measurements quickly become completely positive. In Fig. 10, the average polarization value of the calculated oscillation is more positive than the measurements, a trend that becomes more prominent as the distance from the resonance increases. The reason for this disagreement is not understood.

Once the resonant frequency and the frequency of the bunching cavity $(750602.5 \pm 0.5 \mathrm{~Hz})$ are known, then it is possible to determine other parameters of the beam and properties of the COSY ring for the setup that existed during this experiment. These are given in Table IV where $p$ is the beam momentum, $C$ is the ring circumference, and $T$ is the beam kinetic energy. All model calculations use these values.

TABLE III. Corrections to the rf-solenoid frequency shifts.

\begin{tabular}{lcc}
\hline \hline $\begin{array}{l}\text { Nominal } \\
\text { shift }(\mathrm{Hz})\end{array}$ & $\begin{array}{c}\text { Additional } \\
\text { shift }(\mathrm{Hz})\end{array}$ & $\begin{array}{c}\text { Oscillation } \\
\text { frequency }(\mathrm{Hz})\end{array}$ \\
\hline-2 & -0.096 & 2.676 \\
-1 & -0.121 & 2.006 \\
+1 & -0.161 & 1.864 \\
+2 & -0.126 & 2.506 \\
Average $-0.13 \pm 0.04$ & & \\
\hline \hline
\end{tabular}

\section{B. Influence of synchrotron oscillations}

Before embarking on a detailed description of the effects of synchrotron oscillations on polarization changes induced with an rf solenoid, it is important to understand how a simple momentum spread affects such processes when the beam is not being bunched. The polarization history for different particles in the beam make up a basis set of functions that, when suitably averaged with weights given by the momentum distribution, describe the results of

TABLE IV. Beam and machine parameters.

\begin{tabular}{c}
\hline \hline Assumed \\
$c=299792458 \mathrm{~m} / \mathrm{s}$ \\
$m_{d}=1875.612793(47) \mathrm{MeV} / \mathrm{c}^{2}$ \\
$G=-0.14298754(26)$ \\
\hline Measured \\
\hline$f_{\mathrm{CYC}}=750602.5(5) \mathrm{Hz}$ \\
$f_{\mathrm{RES}}=871434.13(4) \mathrm{Hz}$ \\
$f_{\mathrm{SYNC}}=331(1) \mathrm{Hz}$ \\
\hline Calculated \\
\hline$p=970.057(24) \mathrm{MeV} / \mathrm{c}$ \\
$C=183.4817(37) \mathrm{m}$ \\
$T=236.006(11) \mathrm{MeV}$ \\
$\beta=1.1258289(58)$ \\
$\beta=0.4593904(89)$ \\
\hline \hline
\end{tabular}


an rf-solenoid induced manipulation of the polarization. When the dominant effect arises from synchrotron oscillations rather than momentum spread, a different set of basis functions becomes appropriate. The difference between these two sets, as well as the difference in their polarization effects, can be used to identify which process is important in a given experimental situation when the beam is bunched and both are possible.

For a coasting beam without bunching, the spread in momentum generates a spread in the spin tunes. If there is an initial horizontal polarization component, then that component will gradually decrease as the spin axes of individual particles in the beam drift away from one another. This spread would also be evident in the response to an rf solenoid operating at a fixed frequency since the solenoid would be on resonance for some parts of the beam and off resonance for others. The result would be a weighted average over curves such as those shown in Figs. 9 and 10. An oscillation would start and then become damped as the components got out of phase with each other. The off-resonant parts would be predominantly positive and lead to a positive average for the result. Examples calculated for various resonance widths in $\Delta p / p$ are shown for a coasting beam in Fig. 11. A slice through such curves for various off-resonance rf-solenoid frequencies at a specific time after the rf-solenoid process has started gives rise to a family of resonance shapes as a function of rf-solenoid frequency as discussed by Morozov [8].

One way to reduce this first-order resonance spread, which gives rise to the decoherence of in-plane polarization, is to bunch the beam, thereby forcing all particles to be on average isochronous (also known as synchrotron phase stability). This eliminates the first order dependence

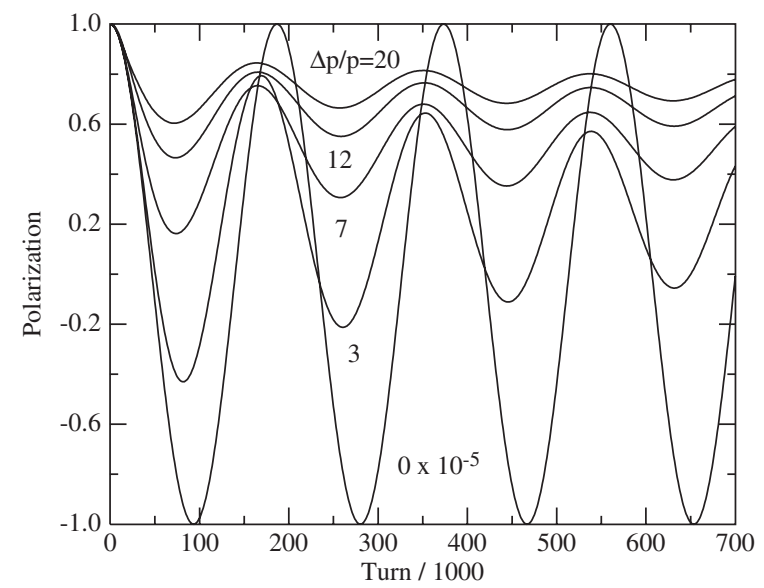

FIG. 11. A variety of time curves showing the vertical polarization oscillations induced by an rf solenoid operating on the $1-G \gamma$ resonance for a coasting beam with a Gaussian distribution of values of $\Delta p / p$. The curves from bottom to top represent responses calculated for a width of $\Delta p / p=$ $0,3,7,12$, and $20 \times 10^{-5}$. of in-plane decoherence on $\Delta p / p$. While a single synchrotron orbit requires many turns to complete, it is fast when compared to the time required for the rf-solenoid polarization oscillations shown in Figs. 9 and 10. For these sorts of measurements, it may be assumed that any polarization effect will be an average over many synchrotron oscillations. Thus, contributions from positive values of $\Delta p / p$ are quickly canceled by their negative counterpart. Any effects that may arise will come only from terms that depend on $(\Delta p / p)^{2}$ or higher orders.

Second-order contributions can also appear due to path lengthening as a function of the size of the horizontal and vertical emittance. The longer path requires a higher average momentum in order to remain isochronous with the cavity rf. That leads directly to a larger value of the relativistic parameter $\gamma$ and a larger value of the spin tune. Thus, responses like those found in Fig. 11 were expected from measurements made with the uncooled beam. Instead, the response for a similar magnet strength is shown in Fig. 12.

In this case the oscillations appear to damp too quickly for the depth of the first oscillation and converge to a value near zero, suggesting that a different mechanism is present. An alternative way to introduce a range of oscillation histories is to use synchrotron oscillations to advance or delay the transit time of parts of the beam through the $\mathrm{rf}$ solenoid. When this happens, these particles do not feel the effect of the same magnetic field as the central particle. Such a time shift effect is enhanced when the rf solenoid operates on a harmonic of the deuteron spin tune, increasing its rate of change. In addition, when the rf cavity is operated on first harmonic, it allows a larger range of synchrotron oscillation amplitudes. So synchrotron oscillations were included in our "no lattice" model, as discussed in Sec. V. Such changes generate a new family of polarization oscillation curves, as shown in Fig. 13.

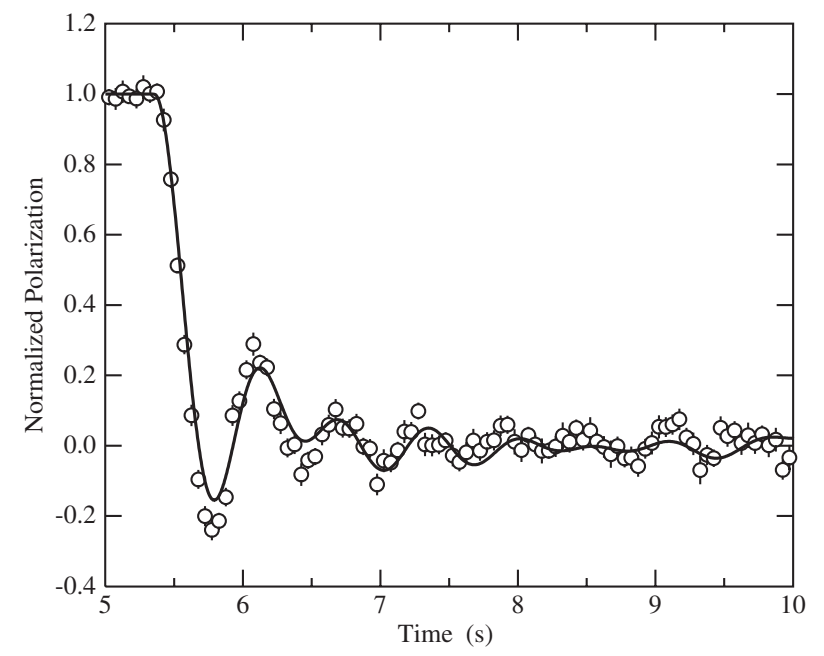

FIG. 12. Measurements made on resonance with the uncooled beam and an rf-solenoid strength of $4.43 \times 10^{-6} \mathrm{rev} / \mathrm{turn}$. 


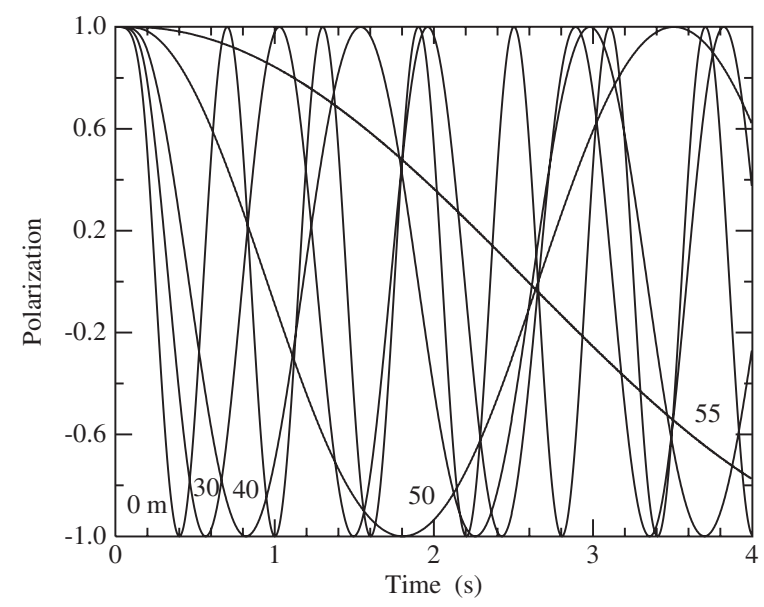

FIG. 13. Sample curves of the change in the vertical polarization as a function of time for different synchrotron amplitudes $A$. All model calculations were made on resonance. As the synchrotron amplitude gets larger, the oscillation period increases. The curves represent values of $A=0,30,40,50$, and $55 \mathrm{~m}$.

All of the curves in Fig. 13 represent the response on resonance. (Moving away from the resonance combines the effect shown here with the trends described earlier.) With larger synchrotron amplitudes, the particle spends less time passing through the rf solenoid at times that are close to optimum for reversing the polarization. Thus, this effectively weakens the solenoid and slows the oscillations for large values of $A$ as appear in the figure.

Aside from the distribution shown in Fig. 6, there is no knowledge from the experiment of the distribution of particles in the beam bunch. It is possible to assume a Gaussian distribution, but this proves to be the wrong shape for a detailed reproduction of measurements such as those in Fig. 12. In order to obtain more flexibility in the model calculation, representative values of $A$ were chosen and the

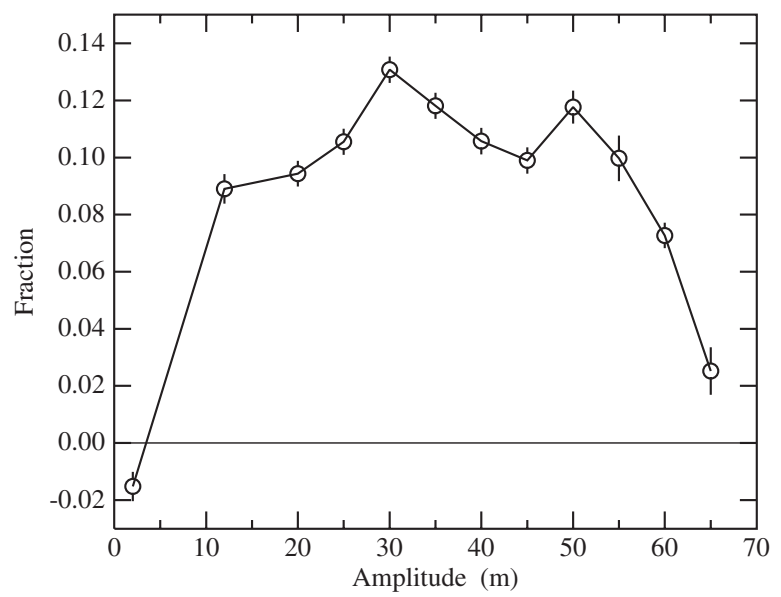

FIG. 14. Distribution of values of $A$ leading to the model calculation of Fig. 12. Note that the first coefficient is negative, an unphysical result. number of particle tracks associated with each value adjusted in order to reproduce the data of Fig. 12. The end result is the model calculation shown in that figure. The fitted fraction of the tracks with different values of $A$ is shown in Fig. 14.

For further calculations, it seemed prudent to replace the fitted distribution of Fig. 14 with a larger number of tracks chosen randomly. Each point of Fig. 14 was replaced by a bin whose area represented the same fraction of the tracks and widths were adjusted to span the range of Fig. 14. The 1000 random tracks were chosen with a distribution of amplitudes that matched the areas of the bins. In the case of the first bin with a negative coefficient, the sign was changed to a positive one to maintain the contribution of small amplitudes to the whole distribution. When this distribution is combined with random synchrotron phases, the resulting beam distribution is shown in Fig. 15. This shape compares favorably with Fig. 6. The FWHM is roughly half of the ring circumference of $183 \mathrm{~m}$ as suggested by Fig. 6 .

This distribution of particles in the beam seems to change by small amounts over time during the experiment. Figures 16 and 17 show time curves calculated with the model of Fig. 12, but with different solenoid strengths. In Fig. 17, agreement is not as good. In particular, more tracks are needed with small amplitudes and faster oscillation frequencies to better match the first two oscillations in the polarization. Adding such tracks improves the agreement for that feature, but this change overestimates the oscillations at larger times.

The calculations shown in Figs. 16 and 17 are similar, but in Fig. 17 the data display different polarization oscillations and the central value is now positive. This supports the conclusion that the distribution of synchrotron

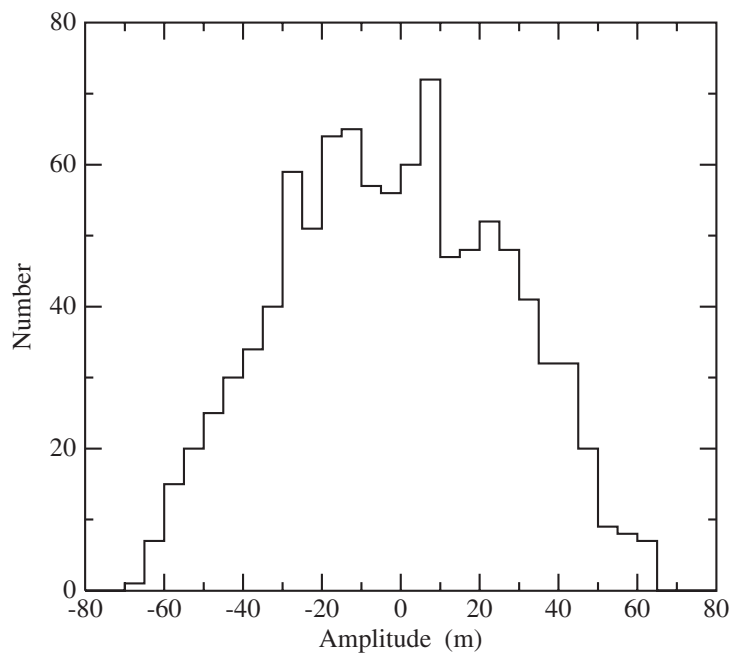

FIG. 15. Distribution of particles in the beam obtained by reproducing the time dependence of Fig. 12 with the amplitude distribution of Fig. 14 and matching that with 1000 tracks following the same amplitude distribution. 


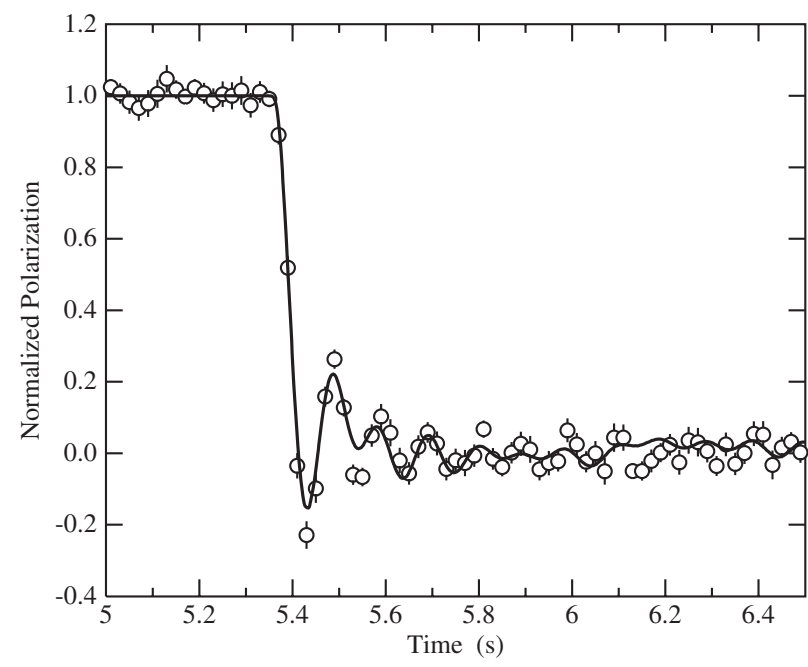

FIG. 16. Measurements similar to Fig. 12 but with a solenoid strength of $2.66 \times 10^{-5} \mathrm{rev} /$ turn. The model calculation uses the distribution of 1000 randomly chosen amplitudes.

amplitudes changes with time. It is possible to reproduce these measurements with a quality similar to that shown in Fig. 12 provided we repeat the fitting process and obtain a new set of coefficients similar to those in Fig. 14. For Fig. 17 the contribution at the larger values of $A$ becomes more prominent, increasing by nearly $50 \%$. During the several hours spent taking these measurements, it is likely that the distribution of particles within the rf bucket changed, even if the bucket potential is unchanged. These measurements are clearly very sensitive to that choice. The range of variation seen in Figs. 16 and 17 demonstrate by example the level of stability of these features.

To summarize, data were taken in two very different situations with continuous monitoring of the polarization

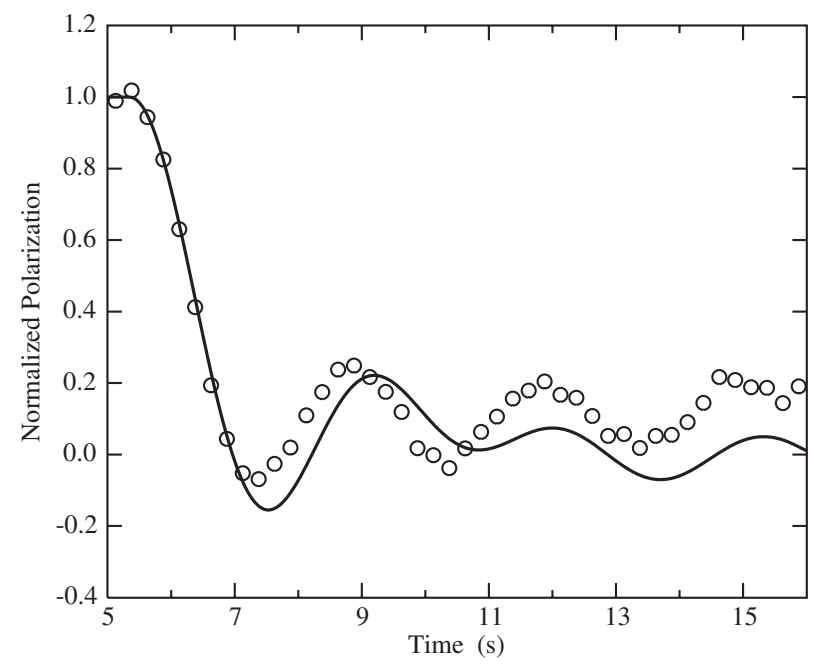

FIG. 17. The same as Fig. 16 but with a solenoid strength of $8.87 \times 10^{-7} \mathrm{rev} / \mathrm{turn}$. The solenoid was turned off at $t=15 \mathrm{~s}$. throughout the beam store, thus providing data with exceptional detail concerning the evolution of the polarization with time. We have considered the case of a bunched, uncooled beam and shown how the damped polarization oscillations seen with the rf solenoid operating at a fixed frequency (and on resonance) depend on the details of the synchrotron oscillation amplitudes. In contrast, the cooled beam had a very small beam bunch size and low momentum spread. In that situation, the application of the $\mathrm{rf}$ solenoid on resonance leads to a very long-lived, slow oscillation of the vertical polarization with essentially constant amplitude. This oscillation responds in a manner seen before [8] to changing the rf-solenoid frequency to an off-resonance value. A comparison of the oscillation frequencies on either side of the resonance permits a very precise determination of the resonant frequency. The tracks of the different particles are sufficiently similar that it is often sufficient to use only the central track as representative of the ensemble, as was done for the calculations in Figs. 9 and 10.

In the case of the uncooled beam, the beam bunch is much larger. This, coupled with the choice to operate the rf solenoid on a harmonic of the spin tune, leads to particles with large synchrotron amplitudes passing through the rf solenoid at nonoptimal times, weakening the effective solenoid strength. The resulting damped polarization oscillations have characteristics that are different from off-resonant behavior. They are particularly sensitive to the detailed distribution of synchrotron amplitudes within the beam bunch. Unfolding this through a fitting process produces a beam bunch distribution that is consistent with what is observed using beam pickup monitors. Because the slower oscillations induced by an rf solenoid operating on resonance still result in polarization oscillations where the polarization completely flips, the features of synchrotron oscillations are distinct from the features of being off resonance.

Next, we will explore some other consequences of the interaction of beam polarization with synchrotron oscillations.

\section{Further exploration of the polarization features of synchrotron oscillations}

In Fig. 9, the polarization oscillations with a cooled beam fall slightly short of reaching either 1 or -1 . Is this an effect of synchrotron oscillations? When presented to a fitting program using the same basis set that was used for the uncooled beam (see Fig. 13), most of the strength appears for amplitudes that are close to zero. The remainder, a few percent, is distributed widely among the larger amplitudes, as shown in Fig. 18. These widely distributed contributions have a shape that is similar to that seen for the uncooled beam, as in Fig. 14. The distribution in Fig. 18 compares well with the distribution measured by the beam pickup shown in Fig. 7, which also has tails emerging on 


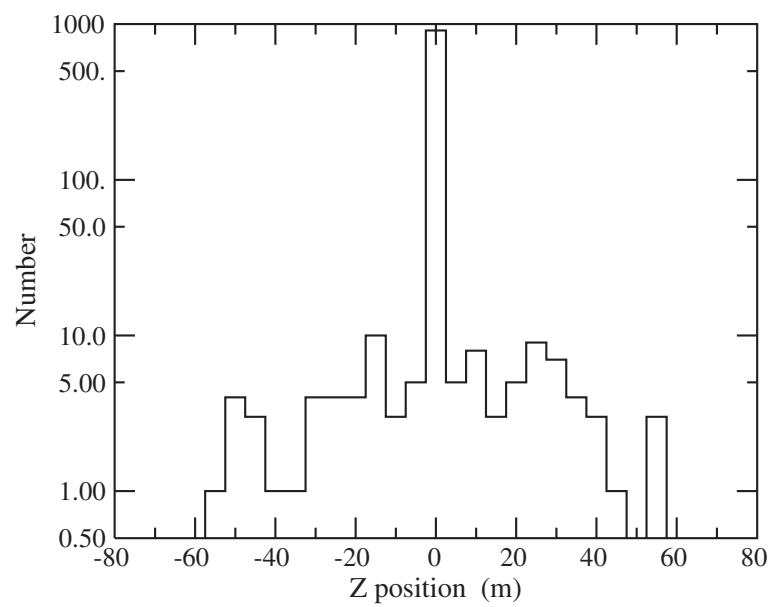

FIG. 18. Like Fig. 15 except for the cooled beam measurements of Fig. 9.

either side of the narrow central peak. The cooling time for the beam is a few tens of seconds. This means that particle collisions with residual gas in the beam pipe or encounters with electrons in the cooling region may remove some particles from the central peak, leading to an uncooled part of the beam that is always being recovered by the cooling system. Eventually this process reaches an equilibrium. In our case, the size of the uncooled component is approximately $9 \%$. The polarization oscillations for these particles are slow and their effect is to reduce the size of the main oscillation in Fig. 9.

In the case of a frequency scan made with the rf solenoid, as illustrated in Fig. 8, particles with particularly large synchrotron amplitudes will respond to the rf solenoid as if it is effectively very weak. At some point these particles will no longer undergo a complete spin flip as the rf-solenoid frequency passes over the position of the resonance. To illustrate this, a model calculation was made of the frequency scan of Fig. 8 using the distribution of amplitudes shown in Fig. 15. Then, for each of the 1000 tracks in that simulation, the vertical projection of the spin was plotted at the end of the frequency scan. The result is shown in Fig. 19. For most of the range of amplitudes $A$, the final spin projection is practically indistinguishable from -1 . At roughly $A=48 \mathrm{~m}$, the effective solenoid strength becomes sufficiently weak that some departure can be seen from a final value of $p_{\mathrm{Y}}=-1$. As the size of $A$ increases, the result moves quickly to +1 , and for higher values falls again toward -1 . Some admixture of the large synchrotron amplitude particles into the beam bunch will thus cause the frequency scan to fall short of a complete polarization reversal. At the point near $A=$ $57.8 \mathrm{~m}$ where the final polarization goes to +1 the period of the oscillation in Fig. 13 goes to infinity. For larger values of $A$ the set of oscillating functions retraces its path back down again with progressively shorter and shorter polarization oscillation periods. Thus $A=57.8 \mathrm{~m}$ is a reflection point in this pattern.

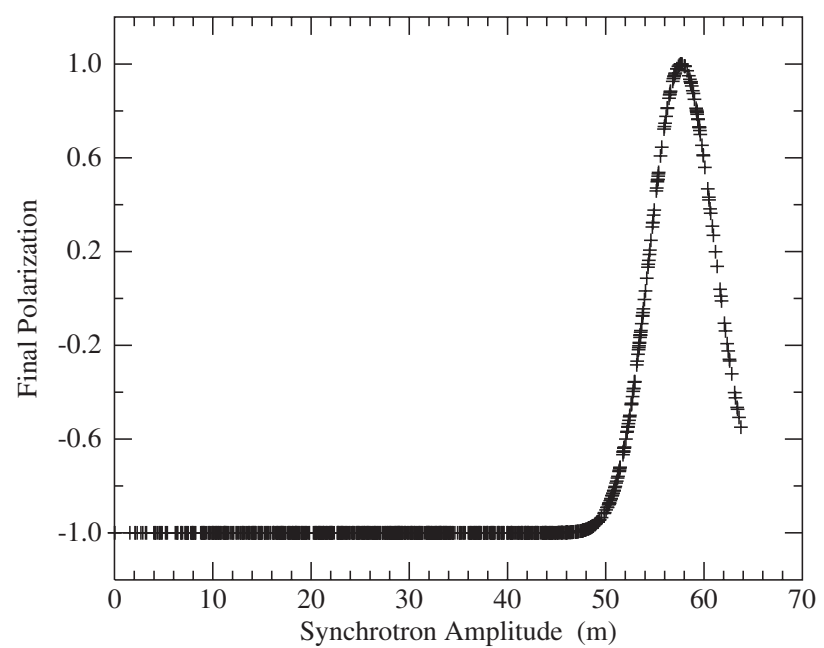

FIG. 19. Final polarization values following a frequency scan as described for the measurements of Fig. 8 displayed here as a function of the synchrotron amplitude parameter $A$ for 1000 tracks.

A calculation based on the sum of the stopping points in Fig. 19 produces a final polarization close to -0.8 while the measurements come close to a final average of -0.96 . A closer reproduction of the measurements of Fig. 8 would require that the fraction of tracks in the critical region of $A>50 \mathrm{~m}$ be reduced by a factor of 5 . The curve in Fig. 8 represents such a rescaled calculation. This sort of variation appears to be typical of the long-term reproducibility of the beams stored in COSY.

\section{Resonance shape}

Using the model described in Sec. V, we matched the predicted oscillation frequencies to the measured ones to better locate the resonance. There are other comparisons to be made, such as the average oscillating polarization. These polarizations as a function of the frequency are shown in Fig. 20 for the cooled data of Figs. 9 and 10 and two additional points further from the resonance. Model calculations with the frequencies adjusted for the average shift in Table III are also shown. In general, these calculations lie above the average polarization values. This is a reflection of the observation that the data points in Figs. 9 and 10 extend below the calculated curves. While the calculation of Fig. 20 was made using only a single particle track with no synchrotron amplitude, the inclusion of a full distribution as in Fig. 18 makes no significant difference $(<0.003)$ in the resulting average polarization.

The width of the calculated resonance curve depends on the values of both the rf-solenoid strength and the time used to ramp-up to full strength at the beginning of the fixed frequency time scan. For these calculations, the strength was $4.43 \times 10^{-6} \mathrm{rev} /$ turn and the ramp-up time was $200 \mathrm{~ms}$. The ramp, created by a signal generator, was linear. The calculated width of the resonance, which is at 


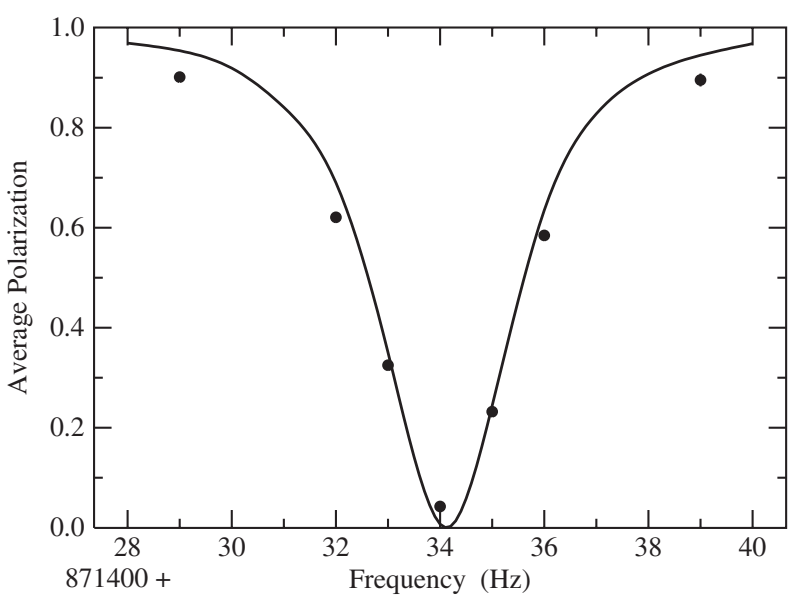

FIG. 20. Measurements of the average oscillating polarization for frequencies on either side of the rf-solenoid spin resonance. The model calculations shown by the curve include the shift of the resonance position from Table III. These measurements were made with cooled beam.

present too narrow, can be made wider if the strength of the solenoid is increased or the ramp-up time is made shorter. Either of these changes would be at variance with the way that the data were acquired.

A series of fixed frequency measurements was made with the uncooled beam. In this case, the full rf-solenoid strength of $2.66 \times 10^{-5} \mathrm{rev} / \mathrm{turn}$ was used. This caused the oscillation pattern to damp quickly, as shown by the example in Fig. 21. So the average polarization was taken to be the mean value after damping for about $0.5 \mathrm{~s}$. The calculation shown in Fig. 21 represents a readjustment of the number of tracks for a selected set of synchrotron amplitudes.

The reproduction of the uncooled resonance data is shown in Fig. 22. The ramp-up time for the rf solenoid

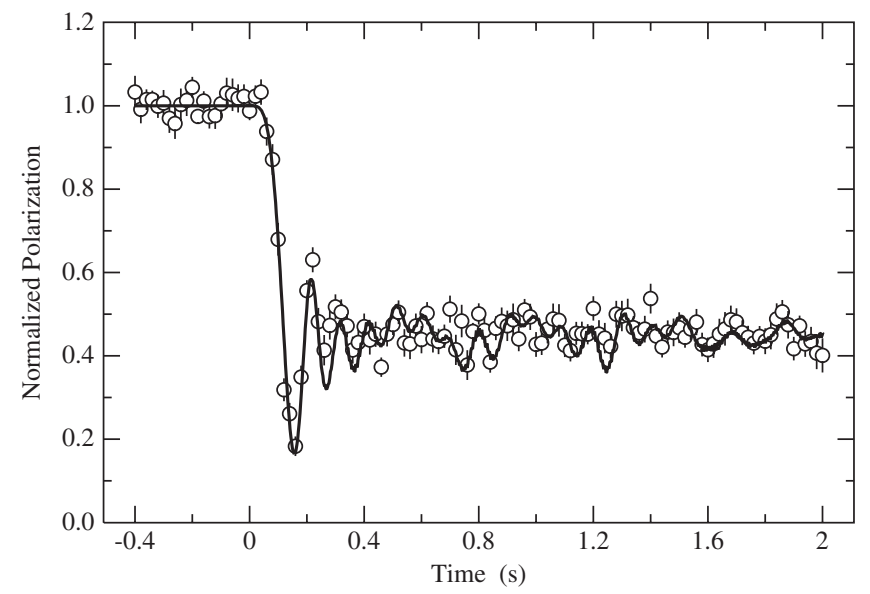

FIG. 21. Measurements off resonance with an uncooled beam. The model calculation was adjusted to reproduce this data by changing the distribution of synchrotron amplitudes. was $200 \mathrm{~ms}$. Here the level of agreement is mixed, with some calculations above their respective data points and others below. The center of the resonance is placed at $871434 \mathrm{~Hz}$. A finer adjustment is not possible because the frequency of the polarization oscillations is not well determined and in any case depends on the distribution of synchrotron amplitudes included in the calculation. Points whose average polarization is less than 0.5 suggest the resonance position should be slightly higher while those with a larger average suggest the opposite.

Besides sensitivity to the solenoid strength and the ramping time, there is also a dependence on the distribution of synchrotron amplitudes. Variations in the trend of the reproduction may reflect changes in the amplitude distribution for the uncooled case from run to run. Each run took about one hour to accumulate.

The shape of the resonance in Fig. 22 appears to be a "V" with gently curving sides. This shape is a consequence of the definition of the average polarization that makes up the data points. If instead the shape is taken to be the polarization at a specific time during the scan or the result at the end of a scan [8], then the shape will be different. On very close inspection, the shape is in fact parabolic at the bottom point of the " $\mathrm{V}$ ". The scale of these graphs is not sufficiently expanded to reveal this feature. That shape is more evident in Fig. 20.

The dependence of the average polarization on the synchrotron amplitude distribution, the rf-solenoid strength, and the ramping time makes these comparisons a strong test of the simple "no lattice" model used here. At the end of the time-on period for the rf solenoid, the solenoid power was ramped to zero. This operation caused the average polarization for the uncooled beam to rise to a more positive value. In general, the model also contained the same feature, but the amount of rise was not well reproduced. Like the effect of the ramp-up time, the

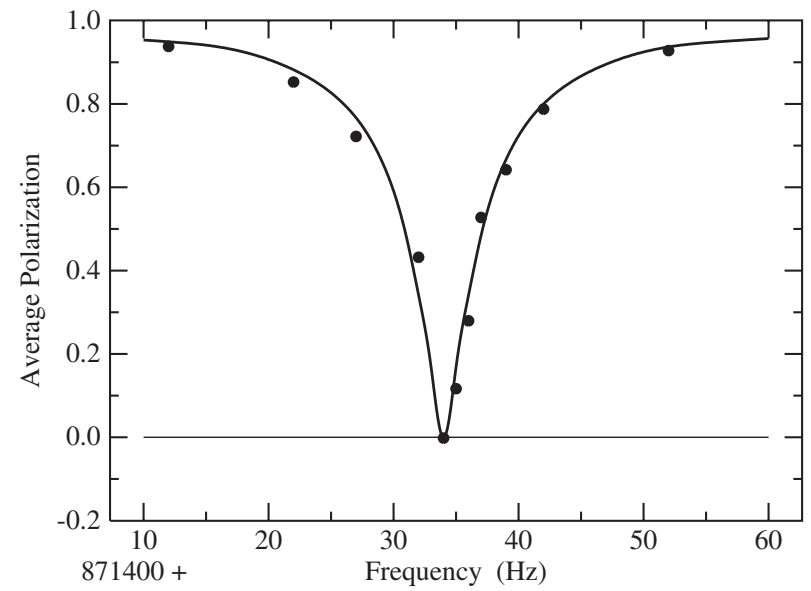

FIG. 22. Data and measurements with an uncooled beam for the average polarization in the vicinity of the resonance as a function of the rf-solenoid frequency. 
amount of rise depended on the ramp-down time. An investigation with different calculated ramp-down times showed that the dependence on this time was exponential, but did not offer any clear clues to the values of the exponential slope nor the final value of the polarization. Such an effect is similar to the dependence of the final polarization in a Froissart-Stora scan with the speed of the scan. A more detailed analysis is needed.

\section{CONCLUSIONS}

This study resulted from the attempt to measure depolarizing effects on the shape of the rf-solenoid spin resonance. Two choices in the setup, the use of a harmonic of the resonance because of the requirements on the operation of the rf solenoid and the choice of bunching the beam on the first harmonic, led to the contribution of large amplitude synchrotron oscillations in the uncooled beam to the evolution in time of the vertical polarization. These contributions contain tracks that reflect an effectively weakened rf solenoid and consequently have a longer oscillation period, even on resonance. For the uncooled beam, this substantially changes the response of the system, creating a damped oscillation in the vertical polarization in the place of the continuously running oscillation observed for the cooled beam. These damped oscillations are built up of precession curves for the individual particles that continue to oscillate between a vertical polarization of +1 and -1 . Compared to the on resonance particle with no synchrotron amplitude, the oscillations in this set of functions have lower frequencies. This makes them different from the oscillations observed by moving the rf-solenoid frequency away from the resonance. In this case, the oscillations away from the resonance are characterized by higher frequencies and smaller amplitudes that cover a maximally positive range. Thus, these two phenomena are distinguishable by the character of the damped oscillation.

The effects of transverse emittance or momentum spread were not directly or unambiguously observed during the experiment. Pursuing this further requires that we look directly at the spin decoherence of a horizontally polarized beam by unfolding the precession of the spin from the polarimeter measurements using time stamps associated with each polarimeter event. Such a stamp would allow us to locate it in the precession history of the beam since the inception of any spin manipulation within the beam store. This study is planned for future experiments.

\section{ACKNOWLEDGMENTS}

The authors wish to thank the staff of COSY for their support during these experiments. We acknowledge helpful discussions with Mei Bai and Yury Orlov. Financial support was received from the German Helmholtz Foundation through funds provided to the virtual institute "Spin and Strong QCD" (VH-VI-231) and the Jülich Center for Hadron Physics (JCHP) located at the Forschungszentrum Jülich GmbH, Jülich, Germany. This publication has also been supported by the European Commission under the 7th Framework Program through the "Research Infrastructures action of the Capacities" Programme (FP7-INFRASTRUCTURES-2012-1, Grant Agreement No. 227431.) This manuscript has been authorized by the Brookhaven Science Associates, LLC under Contract No. DE-AC02-98CH10886 with the U.S. Department of Energy.

[1] F. J. M. Farley, K. Jungmann, J. P. Miller, W. M. Morse, Y. F. Orlov, B. L. Roberts, Y. K. Semertzidis, A. Silenko, and E. J. Stephenson, Phys. Rev. Lett. 93, 052001 (2004).

[2] B. Bonin et al., Nucl. Instrum. Methods Phys. Res., Sect. A 288, 379 (1990); 288, 389 (1990).

[3] V.P. Ladygin et al., Nucl. Instrum. Methods Phys. Res., Sect. A 404, 129 (1998).

[4] D. Albers et al., Eur. Phys. J. A 22, 125 (2004).

[5] J. Bisplinghoff et al., Nucl. Instrum. Methods Phys. Res., Sect. A 329, 151 (1993).

[6] R. Maier, Nucl. Instrum. Methods Phys. Res., Sect. A 390, 1 (1997).

[7] N. P. M. Brantjes et al., Nucl. Instrum. Methods Phys. Res., Sect. A 664, 49 (2012).

[8] V. S. Morozov, A. W. Chao, A. D. Krisch, M. A. Leonova, R.S. Raymond, D. W. Sivers, V.K. Wong, and A.M. Kondratenko, Phys. Rev. Lett. 103, 144801 (2009).

[9] P. D. Eversheim et al., AIP Conf. Proc. 339, 668 (1995); R. Weidmann et al., Rev. Sci. Instrum. 67, 1357 (1996).

[10] S. Kato, N. Matsuoka, T. Noro, T. Saito, H. Sakai, M. Nakamura, M. Yosoi, T. Ichihara, K. Hatanaka, and H. Ogawa, Nucl. Instrum. Methods Phys. Res., Sect. A 238, 453 (1985).

[11] G. Noid (private communication).

[12] Y. Satou et al., Phys. Lett. B 549, 307 (2002).

[13] Gerald G. Ohlsen and P. W. Keaton, Jr., Nucl. Instrum. Methods 109, 41 (1973).

[14] M. Froissart and R. Stora, Nucl. Instrum. Methods 7, 297 (1960). 